\title{
Optimized Fault Tolerance in Distributed Environment
}

\author{
Amanpreet Kaur \\ Department of Computer Science \\ Lovely Professional Univeristy \\ Phagwara
}

\author{
Tejinder Thind \\ Department of Computer Science \\ Lovely Professional University \\ Phagwara
}

\begin{abstract}
VANETs are distributed and self organizing communication networks built up from travelling vehicles. In this research, we have considered controlled latency process by managing the time span between updates for fault tolerance and finding alternative route for vehicles. Delay shown in proposed work is very less than shown in previous techniques due to time management in alternative route selection concept in vehicular adhoc network.
\end{abstract}

\section{Keywords}

Fault Tolerance, Distributed Systems, MANET, Packet Delivery Ratio, Overhead, Delay.

\section{INTRODUCTION}

Vehicular Ad-hoc Networks represent a rapidly emerging and particularly challenging class of Mobile Ad Hoc Networks (MANETs). VANETs are distributed and self organizing communication networks built up from travelling vehicles. These are characterized by very high speed and limited degrees of freedom in nodes movement patterns. Such particular features often make standard networking protocols inefficient or unusable in VANETs. So it is combined with the huge impact that the deployment of VANET technologies could have on the automotive market that explains the growing effort in the development of communication protocols. It is crucial to test and evaluate protocol implementations in real test bed environments, logistic difficulties, and economic issues. Technology limitations make simulation the mean of choice in the validation of networking protocols for VANETs that is a widely adopted first step in development of real world technologies. A critical aspect in a simulation study of VANETs is the need for a mobility model which reflects, as close as possible, the real behavior of vehicular traffic. When dealing with vehicular mobility modeling, we distinguish it in macro-mobility and micro-mobility descriptions [1]. For macro-mobility, we intend all the macroscopic aspects which influence vehicular traffic: the road topology, constraining cars movement, the per-road characterization defining speed limits, number of lanes, overtaking and safety rules over each street of the aforementioned topology, or the traffic signs description establishing the intersections crossing rules. Micro-mobility instead refers to the driver's individual behavior, when interacting with other drivers or with the road infrastructure: traveling speed in different traffic conditions; acceleration, deceleration and overtaking criteria, behavior in presence of road intersections and traffic signs, general driving attitude related to driver's age, sex or mood, etc. It would be desirable for a trustworthy VANETs simulation that both macromobility and micro-mobility descriptions be jointly considered in modeling vehicular movements. Indeed, many non-specific mobility models employed in VANETs simulations ignore these guidelines, and thus fail to reproduce irregular aspects of vehicular motion, such as car acceleration and deceleration in presence of nearby vehicles, queuing at road intersections, clustering caused by semaphores, vehicular congestion and traffic jams as shown in Fig 1 .

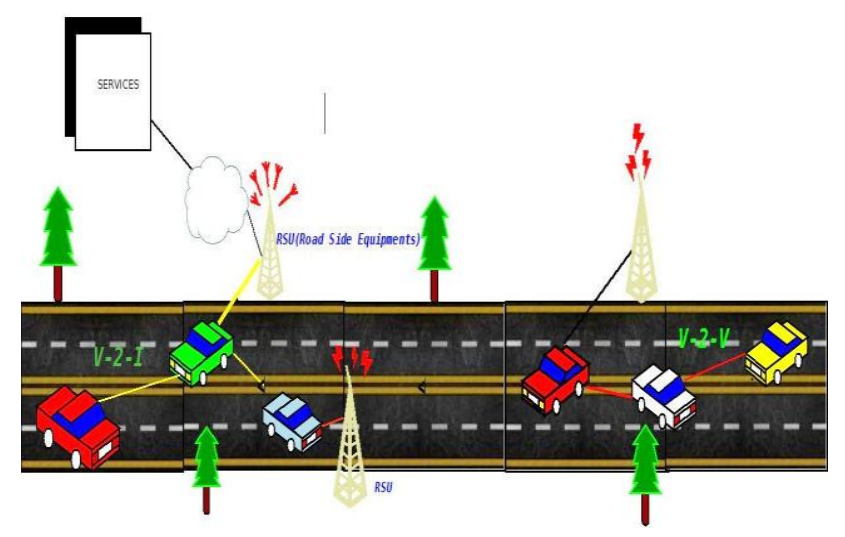

Figure 1: Vehicular Adhoc Network

\section{REVIEW OF LITERATURE}

Since the year of $90 \mathrm{~s}$, the numbers of studies have been carried out to make a Distributed Mobile System Faults Tolerant and Conflict free by proposing many Algorithms. Most of the Research work in Distributed Mobile System has been done towards enhancing and making the system in such a way that it can Tolerate the Faults and Works correctly even in the presence of the Faults.

Shivani Jain, HimanshuTyagi, Charu Gupta presented a work on Secure and fault Tolerance handoff in VANET using special mobile agent [2]. In this threshold based optimal access CAC have been implemented which provides better handoff calls. Mobile agent is assigned to each vehicular node which provides better handoff. Mobile agent moves from the range of one vehicular node to that of another vehicular node and mobile agent is used when handoff is required. Check pointing and replication system is used for retaining reliability. Duplicate copy of data is available at each vehicular node which provide backup of calls and hence provide good fault tolerance.

Tejinder Thind, Rachit Garg, Uminder Kaur and Dinesh Kumar proposed the Paradigms in Fault Tolerant Check pointing Protocols in Distributed Mobile Systems [3]. Nowadays the system are being designed with number of processors and more powerful systems because of advancement of technology and rising demand of society is being changing. The system with number of processors must be able to work smoothly with fault tolerant system but the major issue is that how to handle and manages the faults. To 
make the system Fault tolerant and to achieve the reliability of the system many techniques have been developed like transaction, group communication and rollback recovery. One of the Most Common method is applied to these kinds of failures is Check pointing Protocol. In this proposed model they to overcome the problem of roll backing by roll back only the crashed processor i.e. instead of roll backing the all processors to earlier stage even if the single processor crashes. In this Proposed model the distribution transaction have been done to make the system fault tolerant by applying the TwoPhase Commit Protocol and Check Pointing Algorithm. With these techniques it provides a system to restore back to consistent state even in the presence of failure.

Masashi Shiraishi, Tomoya Enokido, and Makoto Takizawa implemented the Fault Tolerant for mobile agents in Distributed Object Systems [4]. Objects are manipulate by the Mobile Agents on the object servers by entering first into the object server where the agent manipulate the objects .After that on the basis of all or some of the object servers decision is made by the agent either commit or abort. They also showed up how to resolve the conflict by applying some negotiations conditions and also based on the types of the agents means is it's an ordered agent or unordered agent. If there is a conflict occur in between the agents in object server than the negotiation is occur between the agents. There is also a Surrogate Agent which holds the all Objects which is manipulated by the Agent. In this Paper, based on the commitment conditions the negotiation is done. They implemented the evaluation for applications of mobile agent based on the transaction systems.

K.Mohammadi and H. Hamidi implemented fault tolerant execution for mobile agents in Distributed Systems [5]. To build up the mobile agent system Execution of the mobile agent is an important issue. As the Execution for the mobile agent must be a reliable. In this Mobile Agent system design offering the user transparent Fault tolerance which can be activated only when the request comes or when the task is in need. To Make a Fault Tolerant Mobile Agent system they uses the Fatomas which is bases on Java.There are two Approaches Place Dependent and Agent Dependent which can use to model the Fault tolerant mobile agents which is being executed in distributed system. In Place Dependent Approach fault tolerant is integrated into the mobile agent whereas in Agent Dependent Fault Tolerance which is provide by some protocol is travel with the agent. They implemented their system in Asynchronous Distributed system. For the Providence of flexible and the adaptive system they change the agent domains dynamically.

By Applying the Robust Operation the Performance Measurement of Fatomas will show the overhead which is being introduced to a non-replicated agent after applying some replication mechanism. As by increases of the stages number and the agent size the overhead is also get increases.

Alapan Chakraborty makes a Safe system For Railway signaling which is Fault- tolerant [6]. As the Ultra Reliability in Railway signaling is an Important Issue because it is being related to the train movement and passenger's movement. Nowadays the Safety and the reliability can easily achieve through the fault tolerant by applying some mechanism like recovery, protection or by redundancy. There are different forms of Redundancies; Redundancy can be done in hardware or in software. Ultra reliability now can easily achieve at very low cost because in the advancement of the Microprocessor or the Digital Technology. The Redundancy of the system cannot achieve the reliability or safety of the system. To make the system redundant fail-safe the right management of the redundancy is also important. In this they apply 3 times hardware redundancy to get the high reliability of the railway signaling.

Mohammad Jalil Piran,G. Rama Murthy,G. Praveen Babu give some overview on Vehicular Ad hoc and sensor networks principles and challenges [7]. In this paper they proposed work on Vehicular ad hoc network by using the GPS (Global Positioning System). This Device works in offline mode as well as in online mode by taking the concern of future to get safe and secure they presented the good approach for Intelligent Transportation system. In this they create a system by utilizing the Wireless Sensor Networks for vehicular networks. They gave some of the new challenges and some aspect of VANET and also explained communication architecture and feasible topology which are applicable in VASNET. They employ the wireless Sensor networks as Vehicular Ad Hoc and Sensor Network, and use the VASNET in short. To check the velocity for each instance of vehicle they used the vehicular nodes. In VANET we have some Base Stations like rescue team, police traffic Station. This Base Station can be mobile or it can be a stationary. As the VASNET provides us wireless communication capability between the stationary nodes and vehicular nodes and it also increase the comfort and safety on the highway roads for the vehicles.

Jiangzhou Wang, Huiling Zhu, Nathan J. Gomes described about the concept for mobile communications in high speed trains in distributed environment [8]. In this they proposed the work on broadband wireless communications provision for high speed train. As with the deployment of the high speed train systems and the providence of broadband wireless communications in this system is becoming very crucial. For this they described the network architecture of HST BWC. They introduced the distributed antenna systems, microcells and two potential cellular architecture .In this they also explained the concept for high speed train system i.e. a moving frequency concept .So, by using this concept the handoff is not at all needed for those which is being in the coverage range of one Central Unit. For long period of time there is no need of handoffs because the one central unit may have the large number of remote antenna units. As, Some of the time the handoff calls are needed when the high speed trains crossed the cell boundary between the 2 Central units and this situation is being occurred very frequently.

Train Operation Information distribution system is proposed by the company KYOSAN. They make Mobile Train Information Distributed (TID) which is launched on mobile terminal.In this maintenance data and also train operation range are collected in this. With the help of Mobile TID we can see the information on trains which are coming on the stations and also the information of the trains which are not reaching on time or getting delay train can be easily accessed .This can also be used to support operations notifications which are going on train and helps the train crews as they can see the operations on trains like ordering of train scheduling which might be changed, cancelled operations and the storage instructions which is distributed on mobile terminals.

Tarek K. Refaat, Mai Hassan, Ramez M. Daoud, Hassanein $\mathrm{H}$. Amer presented the work on mixed control traffic by implementing the Ethernet and makes the Fault Tolerant System in train. They integrate the train wagons entertainment on board and the control system [9]. On the Top of Gigabit Ethernet, the entertainment loads and the control system are implemented with each server and controller. Using mixture 
of different periods of the samples make the control load more realistic. Video Streaming and Access of Wi-Fi Internet is means of Entertainment load. In train model entertainment is described as video screens quality, number of Wi-Fi nodes and number of streams. Due to Failure on a single controller when the load get increases the numbers of supported streams get reduced. It reduces the limit of possible number of video which is playing but with one advantage is that this it doesn't reduce the capability of watching video by the passengers. The model which they make is works for Fault Tolerant system as well as for the Fault free system.

S. Ramesh, S. Gobinathan presented a work on how to avoid the collision occurs between the rails and the avoidance of detection of cracks [10]. For the Detection of Cracks the Infra Red rays have been used with Infra Red transmitter and Receiver. The Signal Lamp with the Infra Red Sensor is connected with the Infra Red Receiver. To the Main node the CAN Controller is connected and via GSM its information is being send .The message is further transmitted to the engine and the station which is nearest. The CAN Controller is connected to IR Receiver. For the Collision Avoidance they fixed the sensors in the train wheels which will transmits the infra red rays in the track. The Same Thing is in the Opposite train. The infra red rays will get collides if on the same track there are two trains a. The train will be stop by the driver when the collision get Occur they will go back to their respective engines and make an alarm and get alerts. By Applying this approach it will helps to detect the cracks and also tells how the collisions in the tracks can be avoided.

Mirabadi, M. A. Sandidzadeh, Mr. Hosseingholian and F. Schmid introduced the performance of the system using both the simulated data and real data which was collected from the Sheffield supertram line [11]. A fault detection capability of the multisensor navigation system is introduced in the work. This research work is basically considering a two or three dimensional navigation system for the railway, while most of the railway operators prefer to look at this system as a one dimensional system. Although redesigning the system in one dimension will need considerable changes in the system, but these changes would be all towards simplification of the system, removing the gyroscopes devices from the system and integrating the GPS in a new manner. The results of this research show the great potential of the multisensor integration approach in solving the train navigation problem, achieving higher accuracy, reliability and integrity as compared to the existing systems.

Min-Seok Kim, Min-Kyu Kim and Jong-Woo presented the architecture and reliability model through the passive, active, hybrid redundancy architecture. The reliability in the train control system is analyzed through changing time using MATLAB [12]. The passive redundancy architecture is based on hiding faults. As the probability of fault occurrence on the majority of voters in the train control system is low, the number of the majority of voters is properly decided by standard value of the reliability. The active redundancy architecture is reconstituted by detecting, isolating and recovering faults. The reliability of the Standby Sparing system is higher than the Duplication with Comparison system. The reliability in the active redundancy architecture is lower than the passive redundancy architecture about ten hours. The hybrid redundancy architecture is possible to hide faults and reconstitute systems. However, the hybrid redundancy architecture is complex and cost fairly much of money. The reliability is the highest in the redundancy architectures. The reliability of the train control system is exclusively analyzed in this thesis. When the train control system is designed, the hardware redundancy architecture is chosen by the reliability, availability, maintenance and safety.

Jaime L loret, Francisco J. Sanchez, Juan R. Diaz and Jose M. Jimenez describes a protocol to improve critical real-time railway control systems. The algorithm designed gives us fast recoveries when railway computers fail down [13]. The protocol permits to manage the railway control system from every computer in the network mixing unicast and multicast messages. Excellent results are obtained limiting critical failures recoveries to less than $50 \mathrm{msec}$. Connections between computers are based on computer's capacity, so every computer sends its sensor data to the most capable computers in the network to provide redundancy. Algorithms implemented converge in less than 50 milliseconds to provide real-time recoveries. The protocol mixes unicast and multicast messages to decrease the bandwidth used. Some parameters such as node ID and C and P parameters are defined to have an autonomous system, the railway technicians could manage them manually. Security and data integrity can also be implemented in this research work.

\section{IMPLEMENTATION}

The proposed research work is focused on the reduction of overhead and increasing throughput with fault tolerance and security in vehicular network. The research analysis is done under fully a network based event simulator Network Simulator 2. The deployment of multi-relay street view for mobile devices in the field is done and channel is assigned to the nodes. The Interfaces on the nodes are equal in the initial assignment which is fixed manually by admin. 20 nodes are generated randomly in the range of $10000 \mathrm{~m} \times 10000 \mathrm{~m}$ plane. The scenario considered for experimentation is shown below in figure 2 .

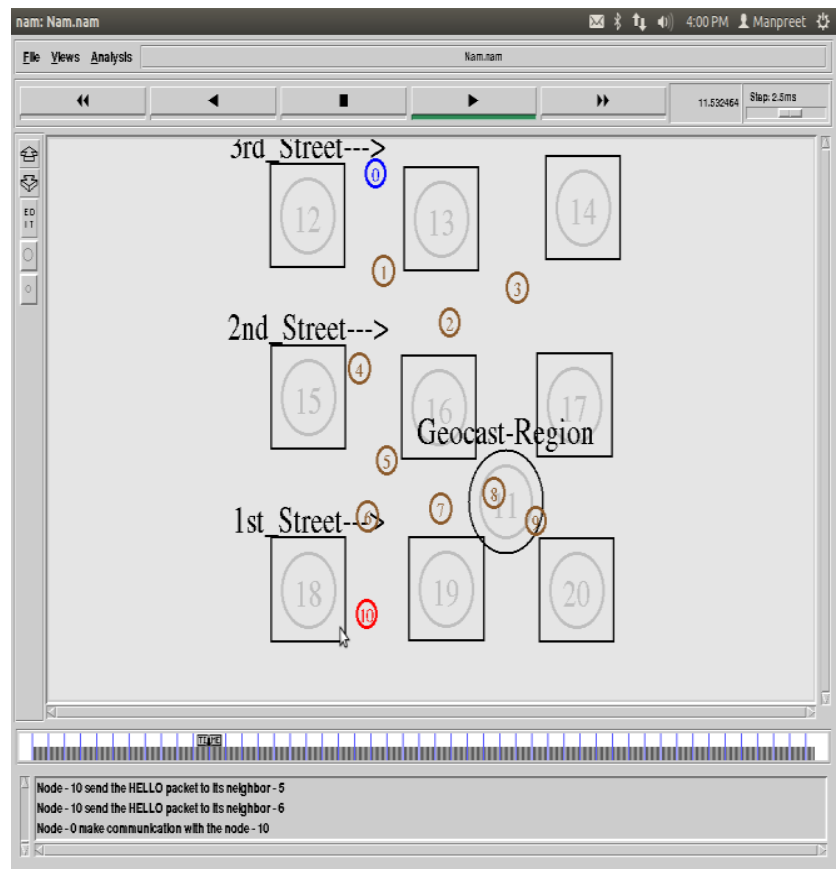

Figure 2: Simulation scenario for experimentation

In multi street network, there is a threshold value for the counter in the whole network which is calculated after selecting change in the network performance. The fixed data rate is generated to 64 Mbps. With this configuration, transmission range without frame error is approximately $50 \mathrm{~m}$ 
and the average number of neighboring nodes is 10 , which shows a moderate density which is shown in figure 3 .

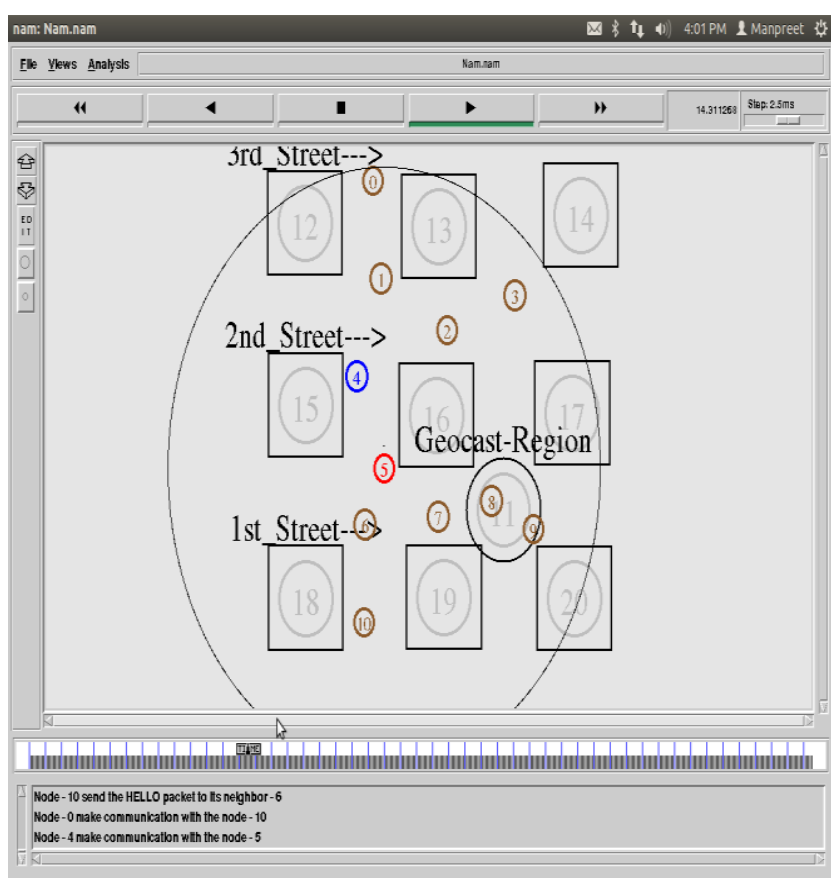

Figure 3: Simulation structural scenario for experimentation

The geocast node is selected which initiate the originator for sending appropriate messages to other vehicles. HLAR protocol is used and added with tolerance of errors when data is sent from one device to other. We proposed a protocol TLAR and compared with Secure-ETX traces of HLAR protocol. Various analyses are obtained by comparing parameters like Packet Delivery Ratio and Overhead, latency and Delay.

A proposed concept of Tolerance protocol communication is used to provide seamless communication in vehicular adhoc network by optimized delay tolerant and error tolerance while sending messages from one vehicle to other and provide efficient scheme to solve the issues created by overhead.

\section{Table 1: Parameters used for the experimentation}

\begin{tabular}{|c|c|}
\hline Parameters & Value \\
\hline Simulator & NS2 \\
\hline Simulation Time & $30 \mathrm{sec}$ \\
\hline No of Subnets & 5 Logical subnets \\
\hline No of Nodes & 20 \\
\hline Traffic Model & \\
\hline Pause Time & CBR \\
\hline
\end{tabular}

\begin{tabular}{|c|c|}
\hline Speed & $64 \mathrm{mps}$ \\
\hline Number of connections & 30 \\
\hline Sub-packet size & 256 bytes \\
\hline Transmit Power & $15 \mathrm{~mW}$ \\
\hline Receiving Power & $13 \mathrm{~mW}$ \\
\hline MAC layer protocol & $802.11 \mathrm{~s}$ \\
\hline Time Slots & Grid Distribution \\
\hline
\end{tabular}

For experimentation we have used network simulator version 2 with animation for the concept of multi vehicle communication with geocast positioning to provide communication for vehicular adhoc networks. Various parameters used for experimentation is shown in above table 1. The detailed experimentation has been considered in the network simulator 2 with optimized delay and error tolerant mechanism as active generator of traffic and measurement of performance of overall network is calculated. In figure 4, concept of the communication between two vehicles is shown.

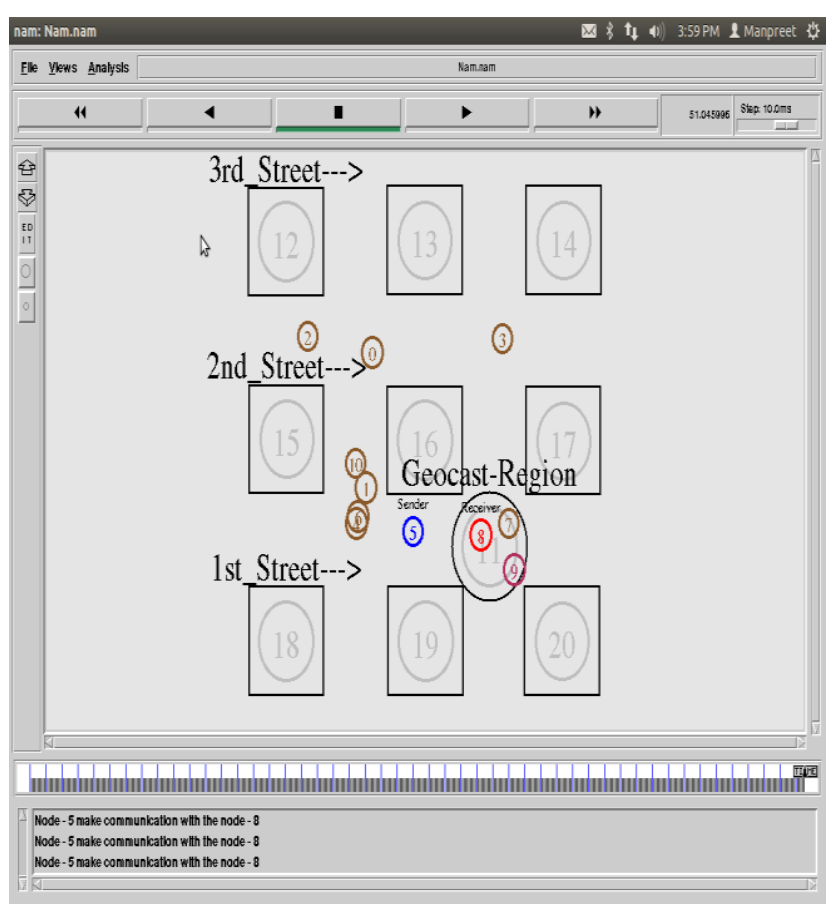

Figure 4: Communication scenario for proposed scheme

\section{RESULTS \\ 4.1 Packet Delivery Ratio}

Packet Delivery Ratio is always used to decide the performance of the network and its processes. So we have considered the experimentation with packet delivery ratio with our proposed scheme. Figure 5, shows the packet delivery ratio analysis for proposed work. In proposed scenario, the complete simulation is first performed with variation of nodes at a distance of $10000 \mathrm{~m}$ with variation of connections. Figure 5, shows the packet delivery ratio for proposed work is $70 \%$ more than HLAR protocol. 


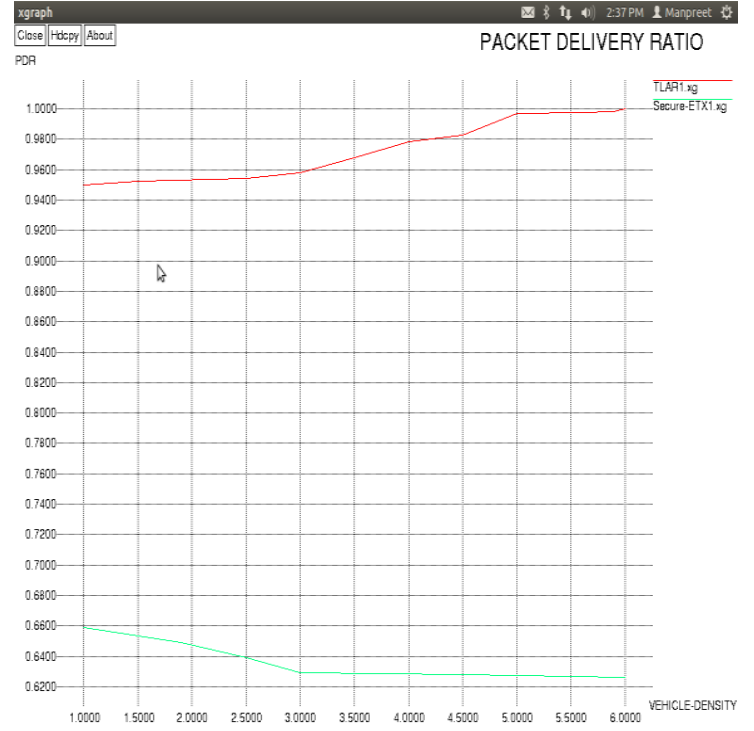

Figure 5: Comparison of optimized TLAR protocol and existing scheme in term of Packet Delivery Ratio

\subsection{Overhead}

Overhead of network is very essential when we carried different processes in judging the behavior and productivity of the network. Figure 6 and Figure 7, showing the overhead of the proposed scheme with vehicle speed of $70 \mathrm{~km} / \mathrm{h}$ and 100 $\mathrm{km} / \mathrm{h}$ respectively.

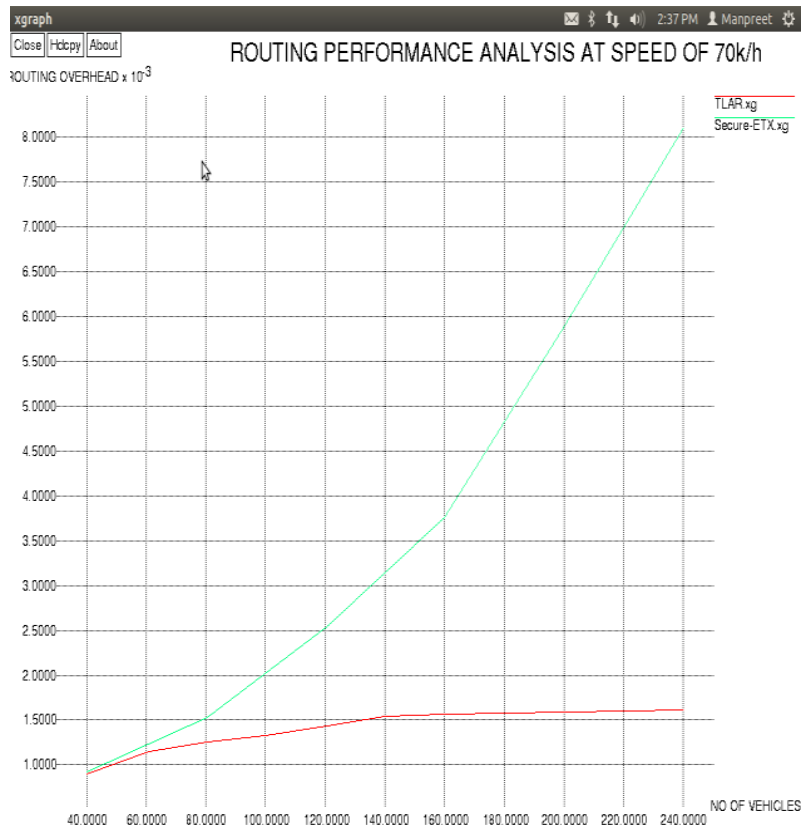

Figure 6: Comparison of optimized TLAR protocol and existing scheme in term of overhead with speed variation

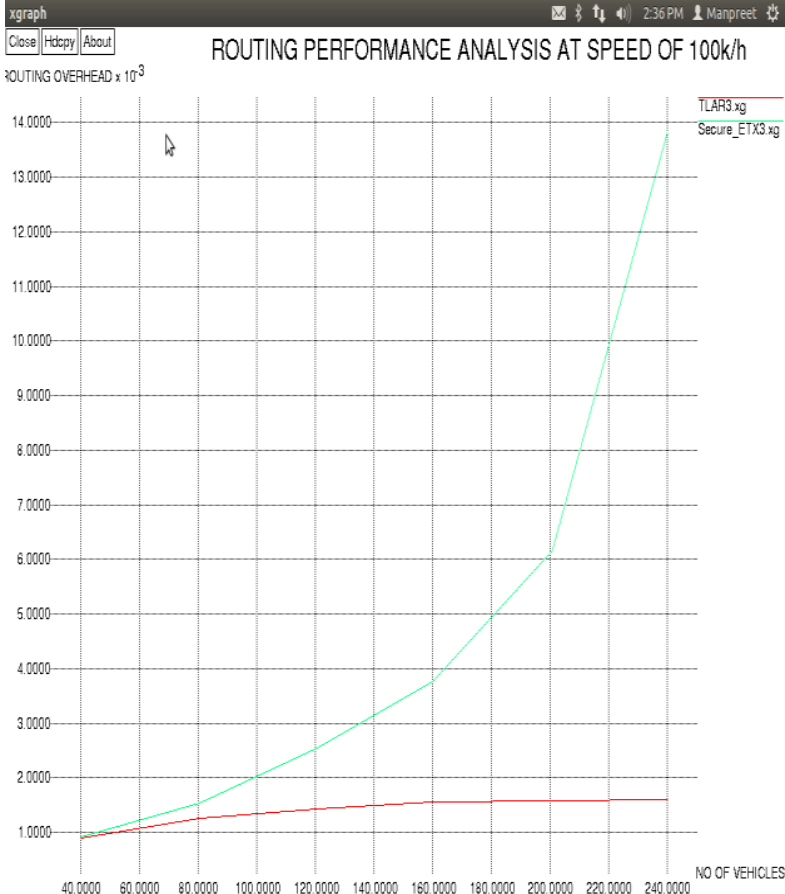

Figure 7: Comparison of optimized TLAR protocol and existing scheme in term of overhead with speed variation

Overhead shown in proposed work is lower than shown in previous techniques such as HLAR due to less delay in sending and receiving packets in network.

So this is clear from figure 5 and figure 6 , that overhead for proposed work is much lesser as compared to already existing protocol. There is $60 \%$ (with $70 \mathrm{~km} / \mathrm{h}$ speed) and $70 \%$ (with $100 \mathrm{~km} / \mathrm{h}$ speed) improvement in overhead in proposed work as compared to HLAR protocol.

\subsection{Delay}

In this research, we have considered controlled latency process by managing the time span between updates for fault tolerance and finding alternative route for vehicles. This process controls the overall latency of the network.

Delay shown in proposed work is very less than shown in previous techniques due to time management in alternative route selection concept in vehicular adhoc network. 


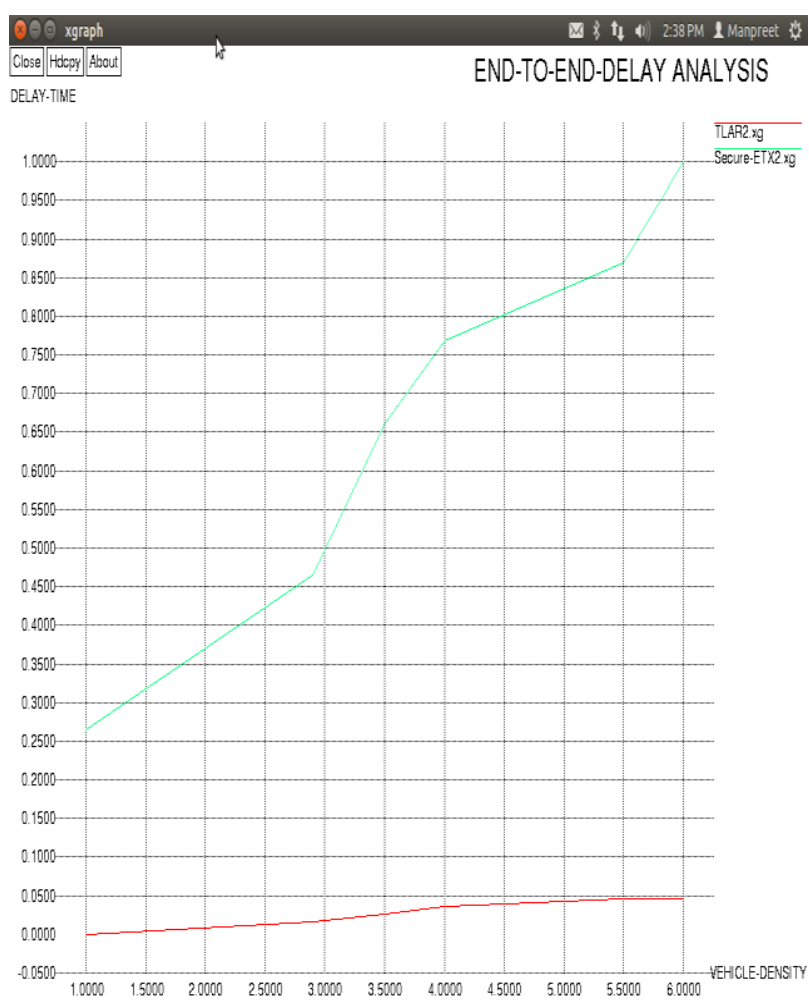

Figure 8: Comparison of optimized TLAR protocol and existing scheme in term of Delay

\section{CONCLUSION AND FUTURE SCOPE}

In VANET, for better and dynamic data communication, a fault tolerance handoff mechanism plays an important role in the handover process between the vehicular nodes during the vehicles are running on the road then mobile agent plays a important role in retrieving the information during the handoff process. Our research gives a mechanism for fault tolerant mobile agent based information retrieval system with security improvements. A method for fault tolerance information retrieval is given in VANET while traffic is moving with random speeds for random time intervals. Special Mobile agents are considered a suitable technology to develop applications such as information retrieval system for mobile computing environment.

\section{REFERENCES}

[1] Marco Fiore, Jerome Harri, "Vehicular Mobility Simulation for VANET", ANSS'07 Proceedings of the $40^{\text {th }}$ Annual Simulation Symposium, Pages 301-309.
[2] Shivani Jain, Himanshu Tyagi, Charu Gupta, "Secure \& Fault”, ICCSEA, SPPR, CSIA, WimoA (NECO), 2013, pp 369-374.

[3] Tejinder Thind, Uminder Kaur, Dinesh Kumar, "Paradigms in Fault Tolerance Check pointing protocols in distributed mobile systems", International Conference on Computing Sciences, IEEE, 2012.

[4] Masashi Shiraishi, Tomoya Enokido, Makoto Takizawa, "Fault Tolerant Mobile Agents in Distributed Objects Systems", Proceedings of the Ninth IEEE Workshop on Future Trends of Distributed Systems, IEEE, 2013.

[5] K.Mohammadi and Hamidi, "Modelling of fault tolerant mobile agents execution in distributed systems", Proceedings of the 2005 systems Communication, IEEE, 2005.

[6] Alapan Chakraborty, "Faulty Tolerant Fail Safe system for Railway Signaling", Proceedings of the world congress on engineering and computer science, San Francisco, USA, October 2009

[7] Mohammad Jalil Piran, Garimella Rama Murthy, G. Praveen Babu, "Vehicular Ad Hoc and Sensor Networks; Principles and Challenges ”, CORR, 2011.

[8] Jiangzhou Wang, Huiling Zhu, Nathan J. Gomes, "Distributed Antenna Systems for Mobile Communications in High Speed Trains", IEEE Journal on Selected Areas in Communications, Vol. 30 No. 4 pp. 675-683, 2012.

[9] Tarek K. Refaat, Esraa A. Makled, Ramez M. Daoud, Hassanein H. Amer, Mai M. Hassan, "Faulttolerant controllers in adjacent wireless networked control systems using 802.11.”, ICUMT, pp. 1-6, 2011.

[10] S. Ramesh, "Detection of Cracks and Railway Collision Avoidance System", 2011.

[11] Mirabadi, M. A. Sandidzadeh, Mr. Hosseingholian, F. Schmid, "Fault Tolerant Train Navigation System".

[12] Min-Seok Kim, Min-Kyu Kim and Jong-Woo, "Reliability Analysis for Train Control System by Hardware Redundancy Architecture in Fault Tolerance System", Journal of International Council on Electrical Engineering Vol. 1, No. 2, pp. 140 144, 2011.

[13] F.J Sanchez Bolumar, J. Lloret Mauri, J.R. Diaz Santos and J.M Jimenez, Herranz, "Standardization and improvement of real time networks technology on railway traffic control system, $7^{\text {th }}$ world congress on railway research, June 2006" 